\title{
The Attentional Effects of Single Cues and Color Singletons on Visual Sensitivity
}

\author{
Alex L. White, \\ Department of Psychology, New York University \\ Rasmus Lunau, and \\ Department of Psychology, University of Copenhagen, København K, Denmark \\ Marisa Carrasco \\ Department of Psychology and Center for Neural Science, New York University
}

\begin{abstract}
Sudden changes in the visual periphery can automatically draw attention to their locations. For example, the brief flash of a single object (a "cue") rapidly enhances contrast sensitivity for subsequent stimuli in its vicinity. Feature singletons (e.g., a red circle among green circles) can also capture attention in a variety of tasks. Here, we evaluate whether a peripheral cue that enhances contrast sensitivity when it appears alone has a similar effect when it appears as a color singleton, with the same stimuli and task. In four experiments we asked observers to report the orientation of a target Gabor stimulus, which was preceded by an uninformative cue array consisting either of a single disk or of 16 disks containing a color or luminance singleton. Accuracy was higher and contrast thresholds lower when the single cue appeared at or near the target's location, compared with farther away. The color singleton also modulated performance but to a lesser degree and only when it appeared exactly at the target's location. Thus, this is the first study to demonstrate that cueing by color singletons, like single cues, can enhance sensory signals at an early stage of processing.
\end{abstract}

\section{Keywords}

vision; attention; attentional capture; single cues; color singletons; feature contrast; contrast sensitivity

\begin{abstract}
Imagine driving on a congested highway in a thunderstorm. In this scene, your retinas are bombarded with light emanating from many objects, some of which are vitally relevant (vehicles ahead, traffic signals) and some of which are inconsequential (bumper stickers, shrubs in the median). By making precise eye movements and "covertly" monitoring objects in the periphery, you can devote your visual system's limited processing resources primarily to those retinal inputs that are relevant to safe driving. This selection of inputs is achieved by the mechanisms of voluntary, or endogenous, visual spatial attention.

You do not have complete control over the distribution of your visual attention, however. Transient changes in the peripheral visual field can automatically capture attention to their location, whether they are relevant—such as a blinking turn signal on the car ahead—or
\end{abstract}

(C) 2013 American Psychological Association

Correspondence concerning this article should be addressed to Alex L. White, Department of Psychology, New York University, 6 Washington Place, New York, NY 10003. alex.white@nyu.edu. 
irrelevant—such as a flash of lightning on the horizon. Such a change can automatically trigger an eye movement toward its location, or, with steady fixation, shift attention covertly in the periphery. For instance, immediately after the lighting flash, you would be better at perceiving details on the horizon near the lightning than on the other side of the road, even if you maintain fixation on the car ahead. This is an example of exogenous covert attention, which is shifted transiently and automatically by salient changes to the retinal image.

The goal of this study was to compare two types of salient events in the periphery-the sudden onset of a single object (a single cue), and the appearance of an array with a single unique element (a color singleton, like a red Ferrari in a line of green minivans) -in terms of their ability to capture attention exogenously to a particular location and modulate low-level visual processing. More specifically, we measured psychophysically whether a color singleton can, like a single cue, enhance processing of a target with no external noise, distractors, or spatial uncertainty, and even when it shares no attributes with the taskrelevant target.

In the laboratory, exogenous spatial attention has been systematically studied using variants of the Posner cueing paradigm (Posner, 1980; reviewed by Carrasco, 2011). Typically, observers begin a trial by fixating at the center of a computer monitor. Then a single small shape, the cue, appears briefly at one of several possible locations on the screen. After a brief delay of $\sim 100 \mathrm{~ms}$, a stimulus array is presented. One of these stimuli (if there are more than one) is the target that observers must respond to. The cue is valid if it had appeared near the target's location, and invalid if it had appeared farther away. Neutral trials, in which the cue appears at fixation or at all locations simultaneously, allow for the assessment of benefits and costs in processing.

In a wide range of perceptual tasks, observers are typically faster and more accurate to respond to the target on valid than on invalid trials (reviewed by Carrasco, 2011). These shifts of attention are involuntary, because they occur even when the cues are completely task-irrelevant and uninformative, and even when they impair performance (e.g., Carrasco, Loula \& Ho, 2006; Yeshurun \& Carrasco, 1998). They also occur faster, and are shorterlived, than voluntary shifts of attention (e.g., Liu, Fuller \& Carrasco, 2006; Müller \& Rabbitt, 1989; Nakayama \& Mackeben, 1989).

Attention can improve vision via a variety of mechanisms, including signal enhancement, external noise exclusion, internal noise reduction, and changes in decision criteria (for a review see Carrasco, 2011). Single exogenous cues may affect performance via both external noise exclusion and signal enhancement (e.g., Lu \& Dosher, 2000). In line with signal enhancement, contrast sensitivity increases at the cued location and decreases at uncued locations (e.g., Carrasco, Penpeci-Talgar \& Eckstein, 2000; Ling \& Carrasco, 2006; Pestilli \& Carrasco, 2005). Correspondingly, neural responses are stronger to validly cued targets than to invalidly cued ones (e.g., Herrmann et al., 2010; Hopfinger \& Mangun, 1998; Li et al., 2008; Liu, Pestilli \& Carrasco, 2005).

In sum, these cueing studies demonstrate that the abrupt onset of a single object in the periphery can capture attention and enhance visual processing. Another pattern of visual input thought to automatically capture attention is local feature contrast. This notion can be traced to studies of visual search, which is easy when the target differs from other elements in the set along only a single feature dimension and is unique in that dimension (such as a red circle among green ones; e.g., Treisman \& Gelade, 1980). This effect is termed "popout" because of the immediate perceptibility of the target stimulus, which remains conspicuous even as more distractor stimuli are added. 
According to saliency-based models of visual selection, "popout" is driven by feature contrast. Computation of salience initially builds on local feature contrast for every feature dimension (e.g., the difference of each image element from its neighbors in terms of color, orientation and motion). This information is represented in separate topographic feature maps that highlight locations where feature values are not homogenous. These maps then converge into a nonspecific saliency map that expresses the overall salience of objects in the visual field. Whatever element carries the strongest salience captures attention of the observer to its location (Itti \& Koch, 2000; Koch \& Ullman, 1985; Nothdurft, 2006; Wolfe, 2007).

In the aforementioned case of popout visual search, the target is a feature singleton. A feature singleton is the only object with a given feature value in a set of objects otherwise homogenous in that feature dimension, and therefore has high visual salience. Feature singletons can seize attentional resources when they are task-irrelevant. For instance, in the "additional singleton" paradigm, participants perform an orientation judgment on a line segment presented inside a target green diamond that appears among several green circles. Response times are slower when one of the circles is red than when all of the shapes are green (Theeuwes, 1991, 1992). This may be because spatial attention is directed toward the most salient object, in this case the singleton red circle, before it reaches the target stimulus. Given that this occurs when the color singleton is completely irrelevant to the task, some authors have concluded that attentional capture is purely stimulusdriven and out of the observer's control (e.g., Giordano, McElree \& Carrasco, 2009; Theeuwes, 1994, 2010; Theeuwes \& Chen, 2005). However, other lines of research have demonstrated attentional capture by feature singletons only when the target shares some attributes with the irrelevant singleton, leading to the conclusion that capture is dependent on the top-down attentional state of the observer (e.g., Bacon \& Egeth, 1994; Becker, Folk \& Remington, 2013; Egeth, Leonard \& Leber, 2010; Folk, Remington \& Johnston, 1992; Folk \& Remington, 1998, 1999; Folk, Remington, \& Wright, 1994; Lamy, Tsal \& Egeth, 2003; Yantis \& Egeth, 1999).

Regardless of whether attentional capture by feature singletons is purely stimulus-driven, the allocation of attention once it is captured resembles, in some ways, covert exogenous capture by single cues. Several studies report that just after the appearance of a color or orientation singleton, responses are speeded to subsequent targets near the singleton and slowed to more distant targets (Burnham \& Neely, 2008; Donk \& Soesman, 2010; Folk et al., 1992; Kim \& Cave, 1999; Mounts, 2000b). Discrimination accuracy is also higher at the singleton's location than others (Joseph \& Optican, 1996; Hsieh, Colas \& Kanwisher, 2011; Mounts, 2000a; Theeuwes \& Chen, 2005), and higher when there is no distracting singleton present compared with when there is one far from the target (Caputo \& Guerra, 1998; Folk, Leber \& Egeth, 2002; Theeuwes, Kramer \& Kingstone, 2004).

The specific mechanism by which singletons alter perception (e.g., signal enhancement, external noise exclusion, uncertainty reduction) is unknown. Furthermore, no study to date has investigated this issue by directly comparing capture by feature singletons to capture by cues that onset alone.

We conducted four experiments to compare these two types of exogenous cues with the same stimuli, task, and observers. The cues themselves were all the same simple shapes that appeared either one at a time or as color singletons in an otherwise homogenous set of similar shapes. We also equated the cue colors in subjective luminance, and counterbalanced the assignment of colors to the singleton or background elements. These measures ensure that any effect of the singleton is in fact attributable to the singleton's feature contrast with the other elements in the set, and not to the singleton by chance having higher local contrast against the background than the other elements. 
Our study specifically investigated whether the appearance of a color singleton-like the appearance of a single cue - enhances subsequent sensory signals arising from its location. In doing so we bring together two extensive lines of research that have seldom overlapped. To measure signal enhancement psychophysically the target stimulus should be presented with no external noise, no distracting stimuli, no local or global masks, and no spatial or temporal uncertainty (Cameron, Tai \& Carrasco, 2002; Carrasco et al., 2000; Carrasco, Williams \& Yeshurun, 2002). We used this approach (in all but one experiment) to determine whether color singleton cues as well as single cues enhance contrast sensitivity. In contrast, previous studies on capture by singletons often present the target in a field of distractors (exceptions: Hsieh, Colas \& Kanwisher, 2011; Kim \& Cave, 1999; Theeuwes et al., 2004), and with much spatial uncertainty regarding the target's location.

All our cues were entirely task-irrelevant and presented briefly before the target, thus likely to elicit only automatic shifts of attention. Furthermore, although our experimental manipulations were not designed to test theories of contingent capture, our color singleton cues had little in common with the task-relevant target (a grayscale grating which was not a singleton). Therefore, any cueing effects would demonstrate that a match between the cue and the observer's top-down set is not strictly necessary for lowlevel capture by irrelevant color singletons, and therefore would constrain theories of attentional capture.

In addressing these issues we also investigated whether the two types of cues differ in the spatial profile of attention that they evoke. The profiles could differ in how far from the cued location the attentional benefit extends. They could also differ in terms of whether, and to what magnitude, there is a region of attentional suppression around the cued location (e.g., Bahcall \& Kowler, 1999; Caputo \& Guerra, 1998; Cutzu \& Tsotsos, 2003; Hopf et al., 2006; Mounts, 2000a, 2000b; Müller \& Kleinschmidt, 2004; Müller, Mollenhauer, Rösler \& Kleinschmidt, 2005; Slotnick, Hopfinger, Klein, \& Sutter, 2002; Steinman, Steinman \& Lehmkuhle, 1995; Turatto \& Galfano, 2001).

We used a simple orientation discrimination task to index attentional effects on visual processing (e.g., Carrasco et al., 2000; Ferneyhough et al., 2013; Herrmann et al., 2010; Pestilli \& Carrasco, 2005). The four experiments differ in the primary measure (thresholds vs. percent correct), the presence of distracting stimuli along with the target, and the distances between the cue and the target. Altogether, the data indicate that color singletons can enhance visual signals, but to a lesser degree and within a narrower spatial region than single cues that onset alone.

\section{Experiment 1A}

\section{Method}

Observers-Ten observers (8 male, ages 19-30) with normal or corrected-to-normal vision participated. Four were graduate students in the NYU Psychology Department and seven were experienced in psychophysical tasks. All observers (but two authors, A.W. and R.L.) were naïve to the purposes of the study.

Apparatus-Stimuli were presented in a darkened room with an Apple Macintosh iMac on a calibrated and linearized CRT monitor (ViewSonic P810) with a refresh rate of $85 \mathrm{~Hz}$ and mean luminance of $45 \mathrm{~cd} / \mathrm{m}^{2}$, viewed at a distance of $57 \mathrm{~cm}$ with a chin rest. Stimuli were programmed in MATLAB (The MathWorks, Natick, MA) using the MGL toolbox (http:// gru.brain.riken.jp/mgl).

Stimuli and procedure-Figure 1 depicts an example trial sequence. Each trial began with a small black fixation cross $(0.3 \times 0.3$ degrees of visual angle $)$ on the center of the 
screen. Two target locations diametrically opposed on a $45^{\circ}$ diagonal 6 degrees of visual angle (dva) in the periphery were each marked with four black dots at the vertices of an imaginary diamond 2.45 dva across. After a variable period of fixation $(500-800 \mathrm{~ms})$, a cue array briefly appeared $(71 \mathrm{~ms})$. The elements of the cue array were disks $0.5 \mathrm{dva}$ in diameter, each $7.75 \mathrm{dva}$ from fixation. On a random half of trials the cue array was a single red or green disk (the single cue condition). In the remaining trials the cue array was a ring of 16 equally spaced, isoeccentric disks, 15 of which were all red or green and one of which was the opposite color (the color singleton cue condition). Color assignment was randomized across trials. The red was created using only the monitor's red gun at full power (CIE $x=0.62, y=0.33$, luminance $=26.9 \mathrm{~cd} / \mathrm{m}^{2}$ ). The green disks used only the green gun, with intensity set by each observer to be subjectively isoluminant with the red using the minimum flicker method (see below). The cue (either the single disk or the color singleton) appeared just outside $\left(1.75^{\circ}\right.$ more eccentric) one of the two potential target locations (randomly intermixed). After a blank interstimulus interval (ISI, $35 \mathrm{~ms}$ ), a Gabor stimulus (4 cycles/dva sinusoidal grating modulated by a Gaussian envelope with 0.28 dva standard deviation, 2 dva total diameter) appeared for $35 \mathrm{~ms}$. Note that the delay between the cue onset and the Gabor offset was too short for saccades to occur.

The Gabor appeared with equal probability in one of the two target locations, either near the cue (on valid trials) or at the opposite location (invalid trials). The Gabor's contrast varied randomly across trials within a set of 9 values equally spaced in logarithmic units from $4.69 \%$ to $80.5 \%$. Immediately after the disappearance of the Gabor, a small black line (the response cue, $0.8 \mathrm{dva}$ long) appeared at fixation, pointing toward the location occupied by the Gabor. This response cue resolved any spatial uncertainty and prompted the observer to report with a keypress whether the Gabor had been tilted left or right of vertical. As soon as the observer responded, the response cue disappeared and an auditory tone indicated whether the response was correct.

Over the course of 51 -hr-long sessions, each observer completed 60 blocks of 112 trials. Two sets of target positions (polar angles of $45^{\circ}$ and $215^{\circ}$ or of $135^{\circ}$ and $315^{\circ}$ ) alternated from block to block. Cue types (single cue vs. color singleton), colors (red vs. green), and validities (valid vs. invalid) were randomly intermixed within blocks with equal probabilities.

At the start of the first 3 sessions observers set the luminance of the green disks to be isoluminant with the red. Observers fixated in the center while a ring of the same 16 disks as used in the main experiment alternated between red and green at $14.2 \mathrm{~Hz}$. By using the up and down arrows, observers adjusted the intensity of the green until the apparent flicker was minimized. The starting intensity was randomized. Adjustments from 4 trials were averaged to acquire the green gun intensity level to be used in the experiment. The intensity from the third session was reused for the remaining sessions.

The first session began with practice trials until the observer could perform the task above chance with a tilt $\$ 40^{\circ}$. Accuracy was emphasized, and the experimenter stressed that the red and green circles (the cues) were irrelevant and uninformative. In the first three sessions, before continuing to the main experiment observers did a pretest to set the difficulty of the task by adjusting the number of degrees by which the Gabor was tilted from vertical. The trial sequence in the pretest ( 2 blocks of 60 trials) was the same as above, except the cue array always consisted of 16 cues all of the same color, and the Gabor's contrast was always $14.06 \%$. The tilt was adjusted with a Quest adaptive staircase (Watson \& Pelli, 1983) to converge on a $75 \%$ correct threshold. Between blocks of the main experiment, if overall performance drifted below $70 \%$ or over $90 \%$, the tilt level was manually adjusted. After the 
first 3 sessions the tilt level from the 3rd session was reused without additional pretests (on average, $7.2^{\circ}$ ).

Data analysis-Trials were excluded with response times (from the onset of the response cue) $\leq 200 \mathrm{~ms}$ or $\geq 5$ standard deviations above the observer's median ( $<1 \%$ of trials excluded on average). In this experiment, and all that follow, we found no differences between cues of different colors (or different polarities, Experiment 1b), and so collapsed them together in the analyses presented here. We fit Weibull curves to the patterns of performance as a function of Gabor contrast (Figure 2A), using the maximum likelihood algorithm provided by the Palamedes Toolbox version 1.5.0 (Prins \& Kingdom, 2009; http:// www.palamedestoolbox.org/). The position ( $\alpha$ ) and lapse rate $(\lambda)$ parameters of each curve were free to vary. We found that slope ( $\beta$ ) did not differ between valid and invalid conditions, so in the analyses presented here, each condition (e.g., singleton cue) was fit with valid and invalid trials collapsed together, and then the slope from that fit was used for both valid and invalid trials fit separately. The same statistical pattern of thresholds was obtained when slopes were free to vary independently. We also note that the risks and benefits of fitting lapse rates are controversial (Wichmann \& Hill, 2001; Prins, 2012). However, we found the same statistical pattern of thresholds when we fixed the upper asymptotes of the curves to the average percent correct at the top 2 contrast levels, similar to a suggestion by Prins (2012).

We evaluated goodness of fit by bootstrapping the fits' deviance scores and testing whether the empirical deviance exceeded a 95\% confidence interval (using the Palamedes toolbox, following Wichmann \& Hill, 2001). From the fits we then extracted $75 \%$ correct contrast thresholds separately for the valid and invalid trials of the single and singleton cue conditions.

Error bars on plots are \pm 1 SEM computed after removing each observer's global mean across conditions, and then scaled by $J /(J-1)$, where $J$ is the number of within-subject conditions in the analysis, following Morey (2008).

\section{Results}

We analyzed contrast thresholds (Figure $2 \mathrm{~B}$ ) with a $2 \times 2$ repeated-measures ANOVA, which revealed a significant interaction between cue type and cue validity: $F(1,9)=18.91$, $p=.002$, as well as significant main effects of cue validity, $F(1,9)=5.72, p=.04$ and cue type, $F(1,9)=7.44, p=.023$. Consistent with the interaction, valid thresholds were significantly lower than invalid thresholds only for the single cue, $t(9)=3.41, p=.008$, but not for the color singleton, $t(9)=0.59, p=.57$. The position (a) parameter of the Weibull fits showed the same statistical pattern, as did overall percent correct.

On average, the fit quality as assessed by $R^{2}$ was high ( $\left.96 \%\right)$. Excluding the few fits (5/40) that were significantly "deviant" $(p<.05)$ yielded the same results: a significant effect of the single cue $(p=0.004)$, but not of the color singleton cue $(p=.58)$ on threshold.

The geometric means of correct response times (RTs; Figure 2C) were also significantly modulated by cue type and validity, with a significant interaction, $F(1,9)=42.5, p=.0001$, as well as main effects of type, $F(1,9)=16.97, p=.003$ and validity, $F(1,9)=117.7, p<$. 0001. RTs were faster on valid than invalid trials with single cues, $t(9)=9.70, p<.0001$, but not with color singleton cues $(t(9)<1, p=.37)$. 


\section{Discussion}

This first experiment clearly demonstrates that the onset of a single cue in the periphery draws attention in a way that increases contrast sensitivity and speeds RTs for subsequent stimuli near that location, compared with the opposite side of fixation. Given that no distracting stimuli, no external noise, and no masks were presented along with the target, and that any spatial and temporal uncertainty about the target was resolved by the postcue immediately after its appearance, we conclude that the single peripheral precue enhanced the magnitude of sensory signals arising from its vicinity (Cameron et al., 2002; Carrasco et al., 2000; Ling \& Carrasco, 2006; Liu et al., 2005; Lu \& Dosher, 2000; Pestilli \& Carrasco, 2005).

In contrast, the onset of an array of items containing a color singleton did not modulate contrast sensitivity or response times in a spatially specific manner, even though the task and stimuli were matched. Curiously, thresholds were low (sensitivity was high) in both valid and invalid color singleton conditions- just as low as in the valid single cue condition. We might have predicted performance with the 16-element cue arrays to be in between the valid and invalid single cue conditions, as if the appearance of cue elements at all locations "distributes" attention roughly equally across space (Barbot, Landy \& Carrasco, 2011, 2012; Carrasco et al., 2002; Talgar, Pelli \& Carrasco, 2004). However, 16 items flashing on the screen may cause a general alerting or arousing affect that improves task performance. Alternatively, the appearance of any item in the periphery could boost sensory signals at its location through a local process that is not influenced by salient events in the rest of the visual field. That could account for the equivalently high sensitivity in the valid single cue, valid singleton and invalid singleton conditions, because in all of those a cue disk flashed at the target location $100 \mathrm{~ms}$ before its appearance. See the General Discussion for further consideration of this issue.

Some authors have argued that feature singletons only capture attention when they are taskrelevant, or have some attributes in common with the task-relevant target-for instance when the target is also a feature singleton (e.g., Egeth et al., 2010; Bacon \& Egeth, 1994; Folk et al., 1992). Based on that literature, a discerning reader could argue that we should not be surprised that our color singleton failed to capture attention.

We must consider another potential reason for the lack of color singleton cueing, however. It is possible that exogenous spatial attention is driven by brief changes in luminance contrast, but not hue (Theeuwes, 1995; Cole, Kentridge \& Heywood, 2005; Lambert, Wells \& Kean, 2003; Lu, 2006; but see Lu \& Zhou, 2005; Snowden, 2002). In Experiment 1A, the single cue always created an isolated, sudden increase in luminance contrast against the local background. In contrast, when the color singleton appeared, there was equal luminance contrast relative to the local background at all 16 locations around the circle, and no luminance contrast across the items in the cue array. Were luminance contrast necessary for a cue to capture attention and modulate contrast sensitivity, this would explain why we found no effect of the color singleton. In the second experiment we tested this possibility by using grayscale cues and singletons defined by contrast polarity (and luminance), rather than hue.

\section{Experiment 1B}

\section{Method}

Observers-Thirteen observers (6 male, ages 19-30) with normal or corrected-to-normal vision participated. Two were graduate students in the NYU Psychology Department and seven were experienced in psychophysical tasks. All observers (but two authors, A.W. and R.L.) were naïve to the purposes of the study. Three had also participated in Experiment 1. 
An additional observer was excluded from analysis because of an inability to learn the task: her final tilt threshold was more than 2 standard deviations above the mean.

Apparatus-Stimuli were presented in a darkened room with an Apple Macintosh iMac on a calibrated and linearized CRT monitor (IBM P260) with a refresh rate of $100 \mathrm{~Hz}$ and mean luminance of $88 \mathrm{~cd} / \mathrm{m}^{2}$, viewed at a distance of $57 \mathrm{~cm}$ with a chin rest.

Stimuli and procedure-All stimuli were the same as in Experiment $1 \mathrm{~A}$ except the cues were achromatic. The cue disks were light $\left(127.7 \mathrm{~cd} / \mathrm{m}^{2}\right)$ and dark $\left(55.06 \mathrm{~cd} / \mathrm{m}^{2}\right)$. On a random half of the trials the cue was a single disk (either light or dark from trial to trial) and on the other half it was a singleton defined by its contrast polarity (light among dark or vice versa). Because the refresh rate was higher on this display, the timing of the stimuli was slightly different: $70 \mathrm{~ms}$ cue, $40 \mathrm{~ms}$ delay, and $40 \mathrm{~ms}$ Gabor. Eight contrast values were used, spaced equally in $\log$ units from $4.69 \%$ to $73.44 \%$. As before, the tilt was adjusted for each observer in pretests using a middle contrast $(15.6 \%)$ to reach $75 \%$ accuracy (average $=$ $\left.7.1^{\circ}\right)$.

\section{Results}

Contrast thresholds (Figure 2B) were affected both by cue validity, $F(1,12)=6.95, p=.022$ and cue type, $F(1,12)=7.35, p=.019$, and there was a significant interaction between them, $F(1,12)=6.23, p=.028$. The single cue significantly lowered thresholds on valid compared to invalid trials, $t(12)=3.06, p=.01$, but the polarity singleton cue did not, $t(12)$ $=0.24, p=.81$.

Excluding the 7 of 52 fits that were significantly "deviant" $(p<.05)$, neither the effect of the single cue nor of the polarity singleton were significant (both $p=>.1$ ). However, we found the same statistical pattern for overall percent correct as for thresholds: interaction between cue type and validity: $F(1,12)=9.80, p=.009)$; effect of single cue validity: $t(12)=4.47, p$ $=.001$; effect of singleton cue validity: $t(12)=0.32, p=.75$. Furthermore, we found the same pattern on percent correct when we only included the middle contrast $(15.6 \%$, the value used to set task difficulty in the pretest): interaction between cue type and validity: $F(1,12)=4.51, p=.055$; effect of single cue validity: $t(12)=3.13, p=.009$; effect of singleton cue validity: $t(12)=0.51, p=.62$. Thus, despite some deviant fits, we conclude that the single cue enhanced contrast sensitivity (mainly by increasing performance at low to midrange contrast levels), but the polarity singleton did not.

RTs (Figure 2C) mirrored the accuracy data: main effect of cue validity: $F(1,12)=60.6, p$ $<.00001$; effect of cue type: $F(1,12)=23.23, p<.001$; interaction: $F(1,12)=80.22, p<$. $00001)$. Single cues lowered RTs on valid trials by an average of $34 \mathrm{~ms}(t(12)=8.51 ; p<$. 00001). The effect of polarity singleton cue on RTs was also significant, $t(12)=3.74 ; p=$. 003, although RTs were lower on valid than invalid trials by an average of only $5 \mathrm{~ms}$.

\section{Discussion}

The pattern of results in Experiment 1B was similar to the pattern in 1A: the single cue captured spatial attention and increased contrast sensitivity at its location, whereas the polarity singleton did not. This occurs whether the singleton is defined by hue or by luminance contrast polarity.

Previous studies demonstrating attentional effects of feature singletons usually present the target along with distractors. ${ }^{1}$ An effect of the cue in a display with distractors could be attributable to changes in how well external noise in the distractors is excluded from the perceptual template, or excluded from the decision-making process, rather than to signal 
enhancement at the target location (e.g., Davis, Kramer \& Graham, 1983; Eckstein, 1998; Eckstein et al., 2009; Foley \& Schwartz, 1998; Kinchla, 1980; Montagna, Pestilli \& Carrasco, 2009; Pestilli \& Carrasco, 2005; Sperling \& Dosher, 1986). It is therefore possible that color singletons capture attention mainly via distractor exclusion.

Accordingly, we conducted Experiment 2 to determine whether our color singleton cues could modulate performance when the target was presented among distractors. Experiment 2 was very similar to Experiment $1 \mathrm{~A}$ except that four gratings appeared simultaneously, one of which was indicated as the target at the end of the trial. Rather than measuring full psychometric functions, we evaluated performance at an intermediate contrast where exogenous attention should have a large effect. In addition, we assessed the relative salience of the two cue types with an independent cue localization task.

\section{Experiment 2}

\section{Method}

Observers-Twelve observers (4 male, ages 19-30) participated in the study. All had normal or corrected-to-normal vision and were naïve to the purposes of the task. None had participated in Experiments 1A or 1B.

Stimuli and procedure-Methodological details were the same as in Experiment 1A, except as indicated below. Figure 3A illustrates the display. The four target locations that were used in alternating pairs in Experiment 1 were all used together on each trial of this experiment, each with the same landmark dots around it. After the cue array and blank delay, 4 Gabor patches appeared simultaneously, one in each of those locations. The Gabors always had the same contrast, either $30 \%$ or $35 \%$, depending on the observer's ability. They were all tilted from vertical by the same magnitude, which was determined by pretest staircases as in Experiment $1\left(M=15.4^{\circ}\right)$, and each Gabor was randomly and independently tilted left or right of vertical. The response cue pointed toward a randomly selected location, designating the Gabor that had appeared there as the target. The observer pressed one of four keys to report its orientation: $z$ or $x$ with the left hand if the target was on the left half of the screen, and < or > with the right hand if the target was on the right half of the screen. Each observer completed 16-17 blocks of 112 trials over two 1-hr sessions.

In addition, at the end of their second sessions, 9 observers completed two blocks of a cue localization task. The display was identical to the main experiment except no Gabors were presented, and no response cue. The task was to press a key with the left hand if the cue (single cue or color singleton) was on the left side of the screen, or a key with the right hand if the cue was on the right side of the screen. Observers were instructed to respond as quickly as possible without sacrificing accuracy.

\section{Results}

Cueing effects-For percent correct (Figure 3B), a $2 \times 4$ repeated-measures ANOVA revealed a main effect of cue type, $F(1,11)=35.82, p<.001$, but no main effect of cuetarget distance $(F(3,33)<1)$, and a marginal interaction $(F(3,33)=2.48, p=0.078)$. Separate one-way ANOVAs for each cue type found no effect of cue-target distance for single cues $(F(3,33)<1)$, but a significant effect for color singletons, $F(3,33)=5.10, p=$. 005 . For the color singleton cue, pairwise comparisons revealed that performance at $0^{\circ}$ cue-

\footnotetext{
${ }^{1}$ There are three exceptions, each of which differs significantly in the timing or measure from our study: one that measured accuracy with no delay between the singleton and target (Theeuwes, Kramer \& Kingstone, 2004); another measured accuracy with a $2250 \mathrm{~ms}$ delay between a subliminal singleton and the target (Hsieh, Colas \& Kanwisher, 2011); and a third that only measured RTs (Kim \& Cave, 1999).
} 
target distance was significantly worse than $90^{\circ}$ away in the same hemifield, $t(11)=3.48, p$ $=.03$, and marginally worse than $90^{\circ}$ away in the opposite hemifield, $t(11)=2.477, p=.09$. Performance at $0^{\circ}$ was also worse than at $-90^{\circ}$ and $90^{\circ}$ collapsed together, $t(11)=3.13, p=$. 01 . Therefore, contrary to our expectation, the color singleton suppressed sensitivity in its vicinity. All pairwise tests were corrected for multiple comparisons by the False Discovery Rate procedure (Benjamini \& Hochberg, 1995), and no other comparisons were significant.

For RTs (Figure 3C), the $2 \times 4$ ANOVA yielded significant main effects of cue type: $F(1$, $11)=70.9, p<.00001$; cue-target distance: $F(3,33)=16.08, p<.00001$; and their interaction: $F(3,33)=20.4, p<.000001)$. For single cues, cue-target distance modulated RTs, $F(3,33)=22.34, p<.00001$, which were faster at $0^{\circ}$ cue-target distance than at the other 3 locations (all ps $<0.001$, corrected). However, for color singleton cues, distance did not modulate RTs, $F(3,33)<1$.

Cue localization task-In the cue localization task, observers were significantly faster to locate the single cue than the color singleton (366 vs. $405 \mathrm{~ms} ; t(8)=16.92, p<.001$ ), and similarly accurate (99.6 vs. $97.8 \%$ correct; $t(8)=1.73, p=0.12)$. The reaction time (RT) data suggest that the single cue was inherently more salient than the color singleton.

\section{Discussion}

This experiment demonstrates that single cues and color singleton cues do not have the same effects on visual performance, and in some surprising ways. First, accuracy was significantly impaired for targets that appeared near the color singleton (while RTs were unaffected). It is as if the color singleton created a ring of suppression around it (up to at least $1.75 \mathrm{dva}$ ). Note this comparison was between distances $0^{\circ}$ and $90^{\circ}$, but that performance near the cue was not significantly different from performance $180^{\circ}(13.75 \mathrm{dva})$ away, which was the only comparison we had in Experiment 1, when we also found no effect.

The single cue failed to significantly modulate orientation discrimination accuracy, unlike in Experiment 1 . However, the single cue did greatly speed responses to nearby targets, more so than in Experiment 1. Although they received the same instructions as in Experiment 1, observers in this experiment may have emphasized speed more than accuracy.

Although perhaps surprising, the suppressive effect of the color singleton is consistent with some previous research: impaired perception (and neural responsiveness) at locations flanking the focus of attention has been reported in a wide range of experimental contexts before. In the selective tuning model ofTsotsos et al. (1995), a "Mexican hat" profile of attentional modulation is a consequence of top-down feedback, and may function to segment the target from the surrounding background. This phenomenon has been reported empirically with both color singletons (Caputo \& Guerra, 1998; Hopf et al., 2006; Mounts, 2000a, 2000b; Turatto \& Galfano, 2001) as well as with single exogenous precues (Cutzu \& Tsotsos, 2003; Mounts, 2000b; Steinman et al., 1995). However, Mounts (2000b) found the ring of suppression around color singletons only when the target was itself a feature singleton, and suppression around a single abrupt onset cue when the target was defined by a conjunction of features. In contrast, we only found evidence for suppression near the color singleton, but not the single cue, and in neither case was the target Gabor a singleton (except on a random $12.5 \%$ of trials, when distractors happened to all be tilted the other way).

In this experiment, we do not have evidence for the full "Mexican hat" profile, only for worse performance near the color singleton cue $\left(0^{\circ}\right.$ polar angle, $\left.1.75 \mathrm{dva}\right)$ than farther away $\left(90^{\circ}\right.$ polar angle, $\left.9.8 \mathrm{dva}\right)$. What we lack is evidence for enhancement at the singleton's exact location, which other studies showing flanking suppression have found (e.g., Mounts, 2000a). In the first two experiments the target never got closer than 1.75 dva to the cue and 
may have fallen partially in a region of suppression, which could explain the null effect of singletons in Experiment 1 (comparing $0^{\circ}$ and $180^{\circ}$ polar angle). In Experiment 3, we measured performance at exactly the cued location. We hypothesized that with this arrangement of stimuli we would find a positive cueing effect of the color singleton.

\section{Experiment 3}

The display in Experiment 3 was similar to Experiment 1 in that only a single Gabor patch was presented on each trial, but similar to Experiment 2 in that there were 4 potential target locations on each trial. The novel aspect was the form of the cues: rather than solid disks slightly more eccentric than the Gabors, they were hollow rings centered at the same eccentricity as the Gabors. This was to avoid forward masking by the cues when they were centered on the target's location (Breitmeyer \& Öğmen, 2006), which could obscure cueing effects (Yeshurun \& Carrasco, 1998; Luck et al., 1996).

\section{Method}

Observers-Sixteen observers (9 male; ages 19-30) with normal or corrected-to-normal vision participated in the experiment. All but one (author A.W.) were naïve as to the purposes of the experiment, and two had participated in Experiment 2.

Stimuli and procedure-Methodological details were the same as in Experiment 2, except as indicated below. The display is depicted in Figure 4A. The cues were hollow rings 0.2 dva thick with an outer diameter of 1.75 dva. They were centered at 6 dva eccentricity where they could fit just inside the landmark dots at target locations. To fit inside the area enclosed by the cues, the Gabors were made slightly smaller than in previous experiments (Gaussian envelope's standard deviation $=0.25 \mathrm{dva}$ ). The placement of the stimuli was like in Experiment 2, except there was only one Gabor present on each trial. We used only one contrast level per observer (25-35\%, depending on their ability in the first practice session).

QUEST staircases in pretest blocks adjusted the tilt magnitude for $80 \%$ correct performance $\left(M=15.37^{\circ}\right)$. Each observer completed 20 blocks of 112 trials over two 1-hr sessions. In addition, at the end of their second sessions, 13 observers completed two blocks of the cue localization task, which was just like that in Experiment 2, but with the ring-shaped cues.

\section{Results}

Cueing effects-A $2 \times 4$ repeated-measures ANOVA on percent correct (Figure 4B) revealed significant main effects of cue type, $F(1,15)=19.90, p=0.0005$, cue-target distance, $F(3,45)=35.70, p<0.00001$, and a significant interaction, $F(3,45)=7.75, p=$ 0.0003 .

For the single cue, cue-target distance significantly affected accuracy, $F(3,45)=33.01, p<$. 00001, which was higher at the cued location than at the other three locations (all $t(15)>6$, all $p$ s $<0.0001$, corrected). Performance at $90^{\circ}$ was marginally higher than at $180^{\circ}, t(15)=$ $2.03, p=0.09$. The color singleton also affected accuracy, $F(3,45)=3.70, p=.018$, which was significantly higher at $0^{\circ}$ than $180^{\circ}, t(15)=4.06, p=.006$. The significant interaction between cue type and cue-target distance reflects the fact that the cueing effect of the color singleton was smaller than that of the single cue. To ensure that this was not an artifact of the overall higher accuracy rates for the color singleton, we applied two transformations to the data: (1) we computed accuracy in units of $d^{\prime}$, which is unbounded; (2) we applied the arcsine-square-root transformation to the proportion correct data, which expands the upper range. Analyses of both transformations revealed the same statistical patterns as described above for proportion correct. 
The RT data (Figure 4C) mirrored the accuracy data, with all effects of the ANOVA significant (cue-target distance: $F(3,45)=37.06, p<.00001$; cue type: $F(1,15)=63.53, p$ $<.00001$; interaction: $F(3,45)=27.57, p<.00001)$. For the single cue, responses were faster at the cued location than at the other three locations, all $t(15)>6$, all $p s<0.0001$, and faster at $-90^{\circ}$ than $180^{\circ}, t(15)=3.05, p=.012$. For the color singleton, responses were faster at the cued location than at the other three locations, all $t(15)>3.5$, all $p \mathrm{~s}<0.01$, but the differences were not as pronounced as those for the single cue.

Cue localization task-In the cue localization task, observers were significantly faster to locate the single cue than the color singleton (367 vs. $413 \mathrm{~ms} ; t(12)=11.21, p<.00001$ ), and marginally more accurate ( 98.8 vs. $97.9 \%$ correct; $t(12)=1.92, p=0.08$ ).

\section{Discussion}

This experiment showed that a color singleton is able to capture attention like a single cue does, increasing visual sensitivity at its retinal location. The key was to place the color singleton cue exactly at the location of the upcoming target-in fact, to have the singleton surround the space the target will occupy. The effect of the color singleton (comparing the cued location to other locations) was reliable but small-much smaller than the effect of the single cue, which was also bolstered with this arrangement of cue and target. The results of the cue localization tasks in Experiments 2 and 3 are consistent with the notion that a uniquely colored item in a large set of items that onset simultaneously is inherently less salient (and attention-capturing) than just one of those items appearing on its own.

Taken together, Experiments 1-3 demonstrate that whereas a single cue enhances sensitivity within its general vicinity up to a few degrees of visual angle away, the color singleton only enhances sensitivity at exactly its location. In Experiments 1A and 1B, there was no difference in sensitivity between locations $1.75 \mathrm{dva}$ and $13.75 \mathrm{dva}$ from the center of the color singleton cue $\left(0^{\circ}\right.$ and $180^{\circ}$ polar angle, respectively). In Experiment 2 , sensitivity 1.75 dva from the color singleton was worse than sensitivity 9.80 dva from the singleton. In Experiment 3, sensitivity 0 dva from the singleton was better than sensitivity 12 dva awaythe only significant positive effect of the color singleton we have obtained so far.

In the fourth and final experiment, we used a finer sampling of cue-target distances to better characterize any differences in the spatial profiles of the attentional shifts triggered by single cues and color singletons.

\section{Experiment 4}

The design of this experiment was much like Experiment 3, except the cues (single cues or color singletons) could appear at any one of 16 locations on each trial. This yielded 9 cuetarget distances (unsigned). We also included two neutral conditions: one in which a single cue appeared around the fixation mark, and one in which all 16 cues appeared and all were of the same color. The neutral conditions allowed us to evaluate the extent to which the cues enhance sensitivity at the cued location, or suppress it at other locations, or both.

\section{Method}

Observers-Fourteen observers (6 male, ages 19-27) with normal or corrected-to-normal vision participated in the experiment. All but one (author A.W.) were naïve as to the purposes of the experiment. Five observers had participated in Experiment 3, and three in Experiment $1 \mathrm{~b}$. 
Stimuli and procedure-The stimuli (Figure 5A) were like in Experiment 3 except that the cue (the single item or the color singleton) could appear at any of the 16 locations around the circle (polar angles from $0^{\circ}$ to $337.5^{\circ}$ in $22.5^{\circ}$ steps). This yielded 9 unsigned cue-target distances. When we divided the data by whether the cue and target were in the same hemifield or not, we found no reliable differences between hemifields, so the analyses presented below use unsigned distances.

We also added two neutral conditions: one with a single cue centered on the fixation mark; the other with all 16 peripheral rings, all of the same color. These cue conditions were equally likely as all the others and intermixed with them. Each observer completed $\sim 40$ blocks of 132 trials in 3 1-hr sessions.

\section{Results}

Accuracy in percent correct (Figure 5B) was modulated by cue-target distance, $F(9,117)=$ $8.52, p<.00001$, cue type, $F(1,13)=75.98, p=.000001$, and their interaction, $F(9,117)=$ $2.97, p=.0032$. Analyzed separately, both the single cue, $F(9,117)=8.52, p<.00001$ and the color singleton cue, $F(9,117)=2.16, p=.030$ significantly affected accuracy. Accuracy in the single cue neutral condition was significantly worse than in the 16-cue neutral condition, $t(13)=3.82, p=.0021$.

To further investigate these patterns of accuracy, for each cue type we conducted two sets of pairwise comparisons: (1) between the respective neutral condition and each of the 9 cuetarget distances; (2) between the $180^{\circ}$ distance and each of the other cue-target distances. The first set identifies locations of enhancement or suppression caused by the cue, and the second tests for significant modulation of sensitivity across space, reflecting both enhancement and suppression, as we did in Experiments 1-3. For each cue type, $p$ values for these 17 comparisons were corrected with the False Discovery Rate procedure (Benjamini \& Hochberg, 1995).

For the single cue, performance differed from the neutral condition only at $0^{\circ}$ distance, $t(13)$ $=4.49, p=.005$. Compared with $180^{\circ}$ away from the single cue, performance was better at $0^{\circ}, t(13)=6.08, p=.0007,22.5^{\circ}, t(13)=3.18, p=.031$, and $45^{\circ}, t(13)=4.10, p=.007$. For the color singleton cue, performance at $0^{\circ}$ was significantly higher than at $180^{\circ}, t(13)=$ $4.01, p=.025$. No other comparison was significant. As in Experiment 3, we found the same pattern of results in units of $d^{\prime}$, or arcsine-square-root transformed proportion correct, which indicate that the smaller cueing effect of the singleton is not due to the overall higher level of performance.

Mean RTs (Figure 5C) were also modulated by cue-target distance, $F(9,117)=16.15, p<$. 00001, cue type, $F(1,13)=113.76, p<.000001$, and their interaction, $F(9,117)=13.49, p$ $<.00001$. Analyzed separately, the single cue affected RTs, $F(9,117)=21.47, p<.00001$, but the color singleton cue did not, $F(9,117)=1.60, p=.124$.

Pairwise comparisons for the single cue revealed that compared with the neutral condition, responses were faster at $0^{\circ}, 22.5^{\circ}$, and $45^{\circ}$, all $t(13)>4.5$, all $p \mathrm{~s}<0.01$. Compared with $180^{\circ}$ from the single cue, response were faster at $0^{\circ}, 22.5^{\circ}, 45^{\circ}, 67.5^{\circ}, 90^{\circ}$ and $112.5^{\circ}$, all $t(13)>2.5$, all $p \mathrm{~s}<0.05)$. Response times were slower in the single cue neutral condition than the 16-cue neutral condition, $t(13)=11.32, p<.00001$. Thus, the RT data qualitatively mirror the accuracy data, with the exception that the color singleton only significantly modulated accuracy and not RTs. There was no sign of any speed-accuracy trade-offs. 


\section{Discussion}

The single cue increased sensitivity in a wide area around it, up to $\sim 5.5 \mathrm{dva}$ from its center. In comparison, the color singleton cue improved sensitivity to a lesser degree and only at its exact location (compared to the location directly opposite fixation). These data confirm the conclusion reached in the previous three experiments.

By comparing with their respective neutral conditions, we found that both cue types enhance sensitivity in their vicinity, but do not suppress sensitivity farther away. This is in contrast to several previous studies with single exogenous cues that measured both benefits and costs in contrast sensitivity relative to a neutral condition (Barbot, Landy \& Carrasco, 2011; Herrmann et al., 2010; Pestilli \& Carrasco, 2005; Pestilli, Viera \& Carrasco, 2007). Furthermore, as in Experiments 1-3, discrimination performance was generally higher when the Gabor was preceded by 16 cue elements than just 1 .

From this experiment alone, it is difficult to determine whether the attentional profiles truly differ in their shapes, and not only in their magnitude. We found no evidence for a region of suppression flanking either type of cue, as we had for the color singletons in Experiment 2. Several differences between the displays in those two experiments could account for the discrepancy: In Experiment 2, we found relatively poor performance for a Gabor positioned in the empty space 1.75 dva from a small disk-shaped color singleton, and the Gabor was presented along with distractors. In Experiment 4, the smallest nonzero distance between the Gabor and the color singleton was $2.34 \mathrm{dva}\left(22.5^{\circ}\right.$ polar angle), and the Gabor appeared on its own, inside an area that had been encircled by another ring-shaped cue element. The lack of flanking suppression in Experiment 4 could be related to any of those differences or to a combination of some of them.

\section{General Discussion}

We compared how spatial attention is automatically drawn to two different types of irrelevant peripheral stimuli: an object that appears on its own (single cue), and a uniquely colored object in an otherwise homogenous array (color singleton cue). We found that both types of exogenous cues enhance subsequent sensory signals at their location, with the same timing and task. However, the color singleton has a weaker spatial cueing effect (in terms of how strongly sensitivity differs between cued and uncued locations) and only enhances sensory signals coming from its precise location. In contrast, the single cue has a robust effect that improves visual performance in a wide region around its location.

In all four experiments we found that overall performance was higher for cue arrays with 16 elements than cue arrays with just 1 element. It may be surprising that performance on invalid color singleton trials was not worse than performance on valid single cue trials, despite the presence of many other potentially distracting transients across the screen. This could be attributable to a general arousing effect of the 16-cue array flashing, or because in the color singleton conditions each location is, in a sense, "cued" by an object's onset.

To further investigate this issue, in Experiment 4 we added neutral cue arrays of 1 and 16 elements to evaluate relative degrees of spatially specific enhancement and suppression. Compared to the 16-cue neutral condition, performance was slightly elevated at the location of the color singleton, and not suppressed at the other locations. Compared with the neutral condition with the single cue at fixation, performance was strongly elevated at the location of the peripheral single cue, and not suppressed at other locations.

These data can be qualitatively summarized as follows: the sudden appearance of any object in the periphery automatically enhances sensitivity at its location, either if it appears alone or 
simultaneously with many other isoeccentric objects. However, an object that is unique gets a small additional boost in its attentional strength. Thus, with singleton cue arrays performance was high for every cue-target distance because each location was stimulated with a cue element. Performance at the exact location of the uniquely colored element was elevated slightly further because of the unique feature contrast that had appeared there.

Our findings that single cues have a greater attentional capture effect (comparing valid to invalid conditions) than color singletons are consistent with several previous studies showing that single cues/abrupt onsets are especially difficult to ignore (e.g., Giordano et al., 2009; Jonides \& Yantis, 1988; Lamy \& Egeth, 2003; Montagna et al., 2009; Pestilli \& Carrasco, 2005; Schreij, Owens, \& Theeuwes, 2008; Yeshurun \& Carrasco, 1998). The results of our cue localization tasks also demonstrate that a color singleton is less conspicuous or less salient than a single item flashing alone. Salience as measured this way, when the cue elements themselves are the targets, seems to correspond to the magnitude of the automatic spatial cueing effects measured in the main experiments.

\section{Signal Enhancement}

The relative benefits in discrimination accuracy we found at the locations of single cues and color singletons are likely to reflect enhancements of sensory signals at early levels of visual processing. This is the first study to demonstrate such a mechanism underlying the attentional effects of salient feature contrast (i.e., color singletons). Other attentional mechanisms may explain effects of color singletons when the targets appeared with simultaneous distractors, external noise, masks, or spatial uncertainty (Belopolsky, Zwaan, Theeuwes, \& Kramer, 2007; Caputo \& Guerra, 1998; Donk \& Soesman, 2010; Folk et al., 2002; Folk et al., 1992; Joseph \& Optican, 1996; Kim \& Cave, 1999; Mounts, 2000a, 2000b; Theeuwes, 1991, 1992, 1994; Theeuwes \& Chen, 2005; Theeuwes et al., 2004).

For instance, some have argued that irrelevant singletons affect performance through higherlevel "filtering costs" (e.g., Becker, 2007; Folk \& Remington, 1998) that slow responses without a spatially specific shift of attention. Such a filtering cost did not play a role in our experiments, as we measured spatially specific modulations of sensitivity. Attentional cueing effects can also be attributable to tuning of the perceptual "filter" (to allow more or less external noise into the decision process) or biased weighting of cued locations in the decision process (Davis et al., 1983; Eckstein et al., 2009; Foley \& Schwartz, 1998; Kinchla, 1980; Lu \& Dosher, 2000; Luck et al., 1996; Shaw, 1984; Sperling \& Dosher, 1986).

External noise exclusion and decision-stage processes cannot explain our results because there was no external noise (save for the distractors in Experiment 2), and there was no spatial uncertainty at the time of the perceptual decision.

Note also that in contrast to many other studies, such as those that use the "additional singleton" paradigm (Theeuwes, 1991, 1992), we briefly flashed our singleton cues $\sim 100 \mathrm{~ms}$ before the target, to be consistent with the single cues. Different mechanisms could come into play when the singleton is presented simultaneously with the target.

\section{Bottom-Up Capture}

In all four experiments, the cues were entirely task-irrelevant and had little in common with the targets, which were never defined as feature singletons. The fact that we were still able to measure effects of the color singleton cues supports the view that attentional capture is purely "bottom-up" or stimulus-driven (e.g., Theeuwes, 2004, 2010; Theeuwes \& Chen, 2005; Theeuwes, Olivers \& Belopolsky, 2010). Models that postulate that capture depends on top-down attentional control would be less likely to predict our findings. 
For example, Folk and colleagues have used modified cueing tasks in which the cue and target stimulus could both either onset alone or appear as feature singletons among other elements (Folk et al., 1992; Folk \& Remington, 1998, 1999; Folk et al., 1994). When an invalid singleton-cue preceded the singleton-target, response times increased. This was also the case when both cue and target appeared alone. However, when cue and target type did not match, there was no attentional capture by the invalid cue, suggesting that capture is contingent on what stimulus attributes are relevant to the observer (Folk et al., 1992; see also Eimer \& Kiss, 2008).

Egeth and colleagues have put forth a related view of top-down control on capture. Bacon and Egeth (1994) distinguished between "modes" of visual search that describe top-down strategies used to find different types of target stimuli. Singleton detection mode is encouraged when the target is a shape or color singleton with an unpredictable feature value (e.g., green or red). In contrast, feature search mode is required when the target has a known feature value but appears among heterogeneous distractors. Several studies have revealed that irrelevant singletons only capture attention in singleton detection mode (e.g., Bacon \& Egeth, 1994; Lamy \& Egeth, 2003; Lamy et al., 2003; Leber \& Egeth, 2006). Lamy and Egeth (2003) found that abrupt onset stimuli (like our single cues) captured attention regardless of search mode, contrary to the contingent capture hypothesis of Folk et al. (1992).

The cueing effects we found in Experiments 3 and 4 with irrelevant singleton cues and nonsingleton targets are inconsistent with strong versions of both of these models of topdown control. However, it is difficult to say which "mode" our observers used. Although the target was not a feature singleton, no feature-selective top-down guidance was needed to locate it among distractors. We are therefore limited in our ability to compare our findings with those of Egeth and colleagues (Bacon \& Egeth, 1994; Lamy \& Egeth, 2003).

Additionally, the fact that single cues failed to significantly modulate accuracy only in Experiment 2, when the target did not also onset on its own, seems to support the "contingent capture" results ofFolk et al. (1992). We note, however, that in this experiment we did find reliable cueing effects on RTs, and that other studies with single exogenous cues have measured strong effects on accuracy when distractors accompany the target (e.g., Carrasco \& Yeshurun, 1998; Giordano et al., 2009; Pestilli \& Carrasco, 2005; Pestilli et al., 2007).

Finally, with regard to top-down control, it is conceivable that our modest effects of color singleton cues would have been bigger if the cues had better matched the observers' topdown attentional "set" or "mode." This would have been the case, for instance, if the target had also been a singleton, or also defined by color. Therefore, top-down factors may at least partially explain why the spatially selective cueing effects of the color singletons were smaller than those of the single cues. However, the fact that even under these conditions the singleton can affect sensory processing constrains contingent capture theories and should be taken as a starting point for further investigations.

\section{Flanking Suppression}

In Experiment 2 we found that performance adjacent to a color singleton is suppressed, compared with locations farther away. Several previous studies using various methodologies demonstrate that perception (e.g., Bahcall \& Kowler, 1999; Caputo \& Guerra, 1998; Cutzu \& Tsotsos, 2003; Mounts, 2000a, 2000b; Müller et al., 2005; Steinman et al., 1995; Turatto \& Galfano, 2001) and neural activity (Hopf et al., 2006; Müller \& Kleinschmidt, 2004; Slotnick et al., 2002) are enhanced at attended locations and suppressed at flanking locations, compared with more distant locations. This has been shown for both single and 
color singleton cues, although we only found such a pattern for singleton cues in Experiment 2 .

In Experiment 4, which was designed to measure the spatial profile of attention in more detail, we did not find such a "Mexican Hat" pattern, although we did find enhancement at the color singleton's exact location (the peak of the sombrero). Comparisons between Experiment 2-in which we did find flanking suppression- and Experiment 4 should be treated with caution, as several factors differed between them (including the cue shape and number of distractors). Either of those could explain why we did not find flanking suppression in both experiments. Alternatively, the spatial extent of flanking of suppression found in Experiment 2 may be smaller than the smallest cue-target distance in Experiment 4. Further work is needed to explore this issue in more detail.

Nonetheless, all together, our data in Experiment 2-suppression close to the color singleton - and in Experiments 3 and 4-enhancement at exactly the singleton's location-are consistent with the spatial profile of attention evoked by a singleton (i.e., a "Mexican hat") as described by Mounts (2000b).

\section{Conclusion}

Attention is rapidly and automatically shifted to particular locations in the peripheral visual field where an object suddenly onsets alone, or where a pattern appears with high local feature contrast. Those exogenous shifts of attention increase contrast sensitivity. Compared with the attentional effects of single cues, however, the effects of color singletons were less pronounced and more constrained to the precise location of the unique element. Nonetheless, both cueing effects allow for more accurate discrimination of subsequent stimuli in regions that in the natural environment would be more frequently relevant for behavior than regions without change across space or time. Comprehensive models of covert attention should encompass findings from these often disparate lines of research, and account for the commonalities and differences between cue types as documented here.

\section{Acknowledgments}

We thank the Carrasco Lab members for helpful comments, and Yilin Zhou for assistance in data collection. This work was funded by National Institutes of Health Grant RO1 EY016200.

\section{References}

Bacon WF, Egeth HE. Overriding stimulus-driven attentional capture. Perception \& Psychophysics. 1994; 55:485-496. [PubMed: 8008550]

Bahcall DO, Kowler E. Attentional interference at small spatial separations. Vision Research. 1999; 39:71-86. [PubMed: 10211397]

Barbot A, Landy MS, Carrasco M. Exogenous attention enhances 2nd-order contrast sensitivity. Vision Research. 2011; 51:1086-1098. [PubMed: 21356228]

Barbot A, Landy MS, Carrasco M. Differential effects of exogenous and endogenous attention on 2ndorder texture contrast sensitivity. Journal of Vision. 2012; 12:1-15.

Becker SI. Irrelevant singletons in pop-out search: Attentional capture or filtering costs? Journal of Experimental Psychology: Human Perception and Performance. 2007; 33:764-787. [PubMed: 17683227]

Becker SI, Folk CL, Remington RW. Attentional capture does not depend on feature similarity, but on target-nontarget relations. Psychological Science. 2013; 24:634-647. [PubMed: 23558547]

Belopolsky AV, Schreij D, Theeuwes J. What is top-down about contingent capture? Attention, Perception, \& Psychophysics. 2010; 72:326-341. 
Belopolsky AV, Zwaan L, Theeuwes J, Kramer AF. The size of an attentional window modulates attentional capture by color singletons. Psychonomic Bulletin \& Review. 2007; 14:934-938. [PubMed: 18087962]

Benjamini Y, Hochberg Y. Controlling the false discovery rate: A practical and powerful approach to multiple testing. Journal of the Royal Statistical Society: Series B (Methodological). 1995; 57:289300.

Breitmeyer, BG.; Öğmen, H. Visual masking: Time slices through conscious and unconscious vision. Oxford, UK: Oxford University Press; 2006.

Burnham BR, Neely JH. A static color discontinuity can capture spatial attention when the target is an abrupt-onset singleton. Journal of Experimental Psychology: Human Perception and Performance. 2008; 34:831-841. [PubMed: 18665729]

Cameron EL, Tai JC, Carrasco M. Covert attention affects the psychometric function of contrast sensitivity. Vision Research. 2002; 42:949-967. [PubMed: 11934448]

Caputo G, Guerra S. Attentional selection by distractor suppression. Vision Research. 1998; 38:669689. [PubMed: 9604099]

Carrasco M. Visual attention: The past 25 years. Vision Research. 2011; 51:1484-1525. [PubMed: 21549742]

Carrasco M, Loula F, Ho YX. How attention enhances spatial resolution: Evidence from selective adaptation to spatial frequency. Perception \& Psychophysics. 2006; 68:1004-1012. [PubMed: 17153194]

Carrasco M, Penpeci-Talgar C, Eckstein M. Spatial covert attention increases contrast sensitivity across the CSF: Support for signal enhancement. Vision Research. 2000; 40:1203-1215. [PubMed: 10788636]

Carrasco M, Williams P, Yeshurun Y. Covert attention increases spatial resolution with or without masks: Support for signal enhancement. Journal of Vision. 2002; 2:467-479. [PubMed: 12678645]

Carrasco M, Yeshurun Y. The contribution of covert attention to the set-size and eccentricity effects in visual search. Journal of Experimental Psychology: Human Perception and Performance. 1998; 24:673-692. [PubMed: 9554103]

Cole GG, Kentridge RW, Heywood Ca. Object onset and parvocellular guidance of attentional allocation. Psychological Science. 2005; 16:270-274. [PubMed: 15828973]

Cutzu F, Tsotsos JK. The selective tuning model of attention: Psychophysical evidence for a suppressive annulus around an attended item. Vision Research. 2003; 43:205-219. [PubMed: 12536142]

Davis ET, Kramer P, Graham N. Uncertainty about spatial frequency, spatial position, or contrast of visual patterns. Perception \& Psychophysics. 1983; 33:20-28. [PubMed: 6844089]

Donk M, Soesman L. Salience is only briefly represented: Evidence from probe-detection performance. Journal of Experimental Psychology: Human Perception and Performance. 2010; 36:286-302. [PubMed: 20364919]

Eckstein MP. The lower visual search efficiency for conjunctions is due to noise and not serial attentional processing. Psychological Science. 1998; 9:111-118.

Eckstein MP, Peterson MF, Pham BT, Droll JA. Statistical decision theory to relate neurons to behavior in the study of covert visual attention. Vision Research. 2009; 49:1097-1128. [PubMed: 19138699]

Egeth HE, Leonard CJ, Leber AB. Why salience is not enough: Reflections on top-down selection in vision. Acta Psychologica. 2010; 135:130-132. [PubMed: 20580341]

Eimer M, Kiss M. Involuntary attentional capture is determined by task set: Evidence from eventrelated brain potentials. Journal of Cognitive Neuroscience. 2008; 20:1423-1433. [PubMed: 18303979]

Ferneyhough E, Kim MK, Phelps EA, Carrasco M. Anxiety modulates the effects of emotion and attention on early vision. Cognition and Emotion. 2013; 27:166-176. [PubMed: 22784014]

Foley JM, Schwartz W. Spatial attention: Effect of position uncertainty and number of distractor patterns on the threshold-versus-contrast function for contrast discrimination. Journal of the Optical Society of America. 1998; 15:1036-1047. 
Folk CL, Leber AB, Egeth HE. Made you blink! Contingent attentional capture produces a spatial blink. Perception \& Psychophysics. 2002; 64:741-753. [PubMed: 12201333]

Folk CL, Remington R. Selectivity in distraction by irrelevant featural singletons: Evidence for two forms of attentional capture. Journal of Experimental Psychology: Human Perception and Performance. 1998; 24:847-858. [PubMed: 9627420]

Folk CL, Remington R. Can new objects override attentional control settings? Perception \& Psychophysics. 1999; 61:727-739. [PubMed: 10370339]

Folk CL, Remington RW, Johnston JC. Involuntary covert orienting is contingent on attentional control settings. Journal of Experimental Psychology: Human Perception and Performance. 1992; 18:1030-1044. [PubMed: 1431742]

Folk CL, Remington RW, Wright JH. The structure of attentional control: Contingent attentional capture by apparent motion, abrupt onset, and color. Journal of Experimental Psychology: Human Perception and Performance. 1994; 20:317-329. [PubMed: 8189195]

Giordano A, McElree B, Carrasco M. On the automaticity and flexibility of covert attention: A speedaccuracy trade-off analysis. Journal of Vision. 2009; 9(3):30, 1-10. [PubMed: 19757969]

Herrmann K, Montaser-Kouhsari L, Carrasco M, Heeger DJ. When size matters: Attention affects performance by contrast or response gain. Nature Neuroscience. 2010; 13:1554-1561.

Hopf JM, Boehler CN, Luck SJ, Tsotsos JK, Heinze H, Schoenfeld MA. Direct neurophysiological evidence for spatial suppression surrounding the focus of attention in vision. PNAS Proceedings of the National Academy of Sciences of the United States of America. 2006; 103:1053-1058.

Hopfinger J, Mangun G. Reflexive attention modulates processing of visual stimuli in human extrastriate cortex. Psychological Science. 1998; 9:441-447.

Hsieh P-J, Colas JT, Kanwisher N. Pop-out without awareness: Unseen feature singletons capture attention only when top-down attention Is available. Psychological Science. 2011; 22:1220-1226. [PubMed: 21852451]

Itti L, Koch C. A saliency-based search mechanism for overt and covert shifts of visual attention. Vision Research. 2000; 40:1489-1506. [PubMed: 10788654]

Jonides J, Yantis S. Uniqueness of abrupt visual onset in capturing attention. Perception \& Psychophysics. 1988; 43:346-354. [PubMed: 3362663]

Joseph JS, Optican LM. Involuntary attentional shifts due to orientation differences. Perception \& Psychophysics. 1996; 58:651-665. [PubMed: 8710445]

Kim M, Cave KR. Top-down and bottom-up attentional control: On the nature of interference from a salient distractor. Perception \& Psychophysics. 1999; 61:1009-1023. [PubMed: 10497423]

Kinchla, RA. The measurement of attention. In: Nickerson, RS., editor. Attention and Performance IX,. Hillsdale, NJ: Erlbaum; 1980.

Koch C, Ullman S. Shifts in selective visual attention: Towards the underlying neural circuitry. Human Neurobiology. 1985; 4:219-227. [PubMed: 3836989]

Lambert A, Wells I, Kean M. Do isoluminant color changes capture attention? Perception \& Psychophysics. 2003; 65:495-507. [PubMed: 12812274]

Lamy D, Egeth HE. Attentional capture in singleton-detection and feature-search modes. Journal of Experimental Psychology: Human Perception and Performance. 2003; 29:1003-1020. [PubMed: 14585019]

Lamy D, Tsal Y, Egeth HE. Does a salient distractor capture attention early in processing? Psychonomic Bulletin \& Review. 2003; 10:621-629. [PubMed: 14620356]

Leber AB, Egeth HE. It's under control: Top-down search strategies can override attentional capture. Psychonomic Bulletin \& Review. 2006; 13(1):132-138. [PubMed: 16724780]

Li X, Lu Z-L, Tjan BS, Dosher BA, Chu W. Blood oxygenation level-dependent contrast response functions identify mechanisms of covert attention in early visual areas. PNAS Proceedings of the National Academy of Sciences of the United States of America. 2008; 105:6202-6207.

Ling S, Carrasco M. Sustained and transient covert attention enhance the signal via different contrast response functions. Vision Research. 2006; 46:1210-1220. [PubMed: 16005931]

Liu T, Fuller S, Carrasco M. Attention alters the appearance of motion coherence. Psychonomic Bulletin \& Review. 2006; 13:1091-1096. [PubMed: 17484441] 
Liu T, Pestilli F, Carrasco M. Transient attention enhances perceptual performance and FMRI response in human visual cortex. Neuron. 2005; 45:469-477. [PubMed: 15694332]

Lu S. Cue duration and parvocellular guidance of visual attention. Psychological Science. 2006; 17:101-102. [PubMed: 16466416]

Lu S, Zhou K. Stimulus-driven attentional capture by equiluminant color change. Psychonomic Bulletin \& Review. 2005; 12:567-572. [PubMed: 16235647]

Lu Z, Dosher BA. Spatial attention: Different mechanisms for central and peripheral temporal precues? Journal of Experimental Psychology: Human Perception and Performance. 2000; 26:1534-1548. [PubMed: 11039483]

Luck SJ, Hillyard SA, Mouloua M, Hawkins HL. Mechanisms of visual-spatial attention: Resource allocation or uncertainty reduction? Journal of Experimental Psychology: Human Perception and Performance. 1996; 22:725-737. [PubMed: 8666960]

Montagna B, Pestilli F, Carrasco M. Attention trades off spatial acuity. Vision Research. 2009; 49:735-745. [PubMed: 19385088]

Morey R. Confidence intervals from normalized data: A correction to Cousineau (2005). Tutorial in Quantitative Methods for Psychology. 2008; 4:61-64.

Mounts J. Attentional capture by abrupt onsets and feature singletons produces inhibitory surrounds. Perception \& Psychophysics. 2000a; 62:1485-1493. [PubMed: 11143458]

Mounts J. Evidence for suppressive mechanisms in attentional selection: Feature singletons produce inhibitory surrounds. Perception \& Psychophysics. 2000b; 62:969-983. [PubMed: 10997043]

Müller HJ, Rabbitt PM. Reflexive and voluntary orienting of visual attention: Time course of activation and resistance to interruption. Journal of Experimental Psychology: Human Perception and Performance. 1989a; 15:315-330. [PubMed: 2525601]

Müller NG, Kleinschmidt A. The attentional "spotlight's” penumbra: Center-surround modulation in striate cortex. Neuroreport. 2004; 15:977-980. [PubMed: 15076718]

Müller NG, Mollenhauer M, Rösler A, Kleinschmidt A. The attentional field has a Mexican hat distribution. Vision Research. 2005; 45:1129-1137. [PubMed: 15707921]

Nakayama K, Mackeben M. Sustained and transient components of focal visual attention. Vision Research. 1989; 29:1631-1647. [PubMed: 2635486]

Nothdurft HC. Salience and target selection in visual search. Visual Cognition. 2006; 14:514-542.

Pestilli F, Carrasco M. Attention enhances contrast sensitivity at cued and impairs it ancued locations. Vision Research. 2005; 45:1867-1875. [PubMed: 15797776]

Pestilli F, Viera G, Carrasco M. How do attention and adaptation affect contrast sensitivity? Journal of Vision. 2007; 7:1-12. [PubMed: 17685805]

Posner M. Orienting of attention. The Quarterly Journal of Experimental Psychology. 1980; 32:3-25. [PubMed: 7367577]

Prins N. The psychometric function: The lapse rate revisited. Journal of Vision. 2012; 12:25-40. [PubMed: 22715196]

Prins, N.; Kingdom, FAA. Palamedes: Matlab routines for analyzing psychophysical data. 2009. http:// www.palamedestoolbox.org

Schreij D, Owens C. Abrupt onsets capture attention independent of top-down control settings. Perception \& Psychophysics. 2008; 70:208-218. [PubMed: 18372744]

Shaw, ML. Division of attention among spatial locations: A fundamental difference between detection of letters and detection of luminance. In: Bouma, H., editor. Attention and performance X. Hillsdale, NJ: Erlbaum; 1984. p. 109-120.

Slotnick SD, Hopfinger JB, Klein SA, Sutter EE. Darkness beyond the light: Attentional inhibition surrounding the classic spotlight. Neuroreport. 2002; 13:773-778. [PubMed: 11997685]

Snowden RJ. Visual attention to color: Parvocellular guidance of attentional resources? Psychological Science. 2002; 13:180-184. [PubMed: 11934005]

Sperling, G.; Dosher, BA. Strategy and optimization in human information processing. In: Boff, KR.; Kaufman, L.; Thomas, JP., editors. Handbook of perception and human performance. Vol. Vol. 1. New York, NY: Wiley; 1986. p. 1-65. 
Steinman BA, Steinman SB, Lehmkuhle S. Visual attention mechanisms show a center-surround organization. Vision Research. 1995; 35:1857-1859.

Talgar C, Pelli D, Carrasco M. Covert attention enhances letter identification without affecting channel tuning. Journal of Vision. 2004; 4:3-31.

Theeuwes J. Cross-dimensional perceptual selectivity. Perception \& Psychophysics. 1991; 50:184193. [PubMed: 1945740]

Theeuwes J. Perceptual selectivity for color and form. Perception \& Psychophysics. 1992; 51:599_ 606. [PubMed: 1620571]

Theeuwes J. Stimulus-driven capture and attentional set: Selective search for color and visual abrupt onsets. Journal of Experimental Psychology: Human Perception and Performance. 1994; 20:799_ 806. [PubMed: 8083635]

Theeuwes J. Abrupt luminance change pops out; abrupt color change does not. Perception \& Psychophysics. 1995; 57:637-644. [PubMed: 7644324]

Theeuwes J. Top-down search strategies cannot override attentional capture. Psychonomic Bulletin \& Review. 2004; 11:65-70. [PubMed: 15116988]

Theeuwes J. Top-down and bottom-up control of visual selection. Acta Psychologica. 2010; 135:7799. [PubMed: 20507828]

Theeuwes J, Chen CYD. Attentional capture and inhibition (of return): the effect on perceptual sensitivity. Perception \& Psychophysics. 2005; 67:1305-1312. [PubMed: 16555583]

Theeuwes J, Kramer AF, Kingstone A. Attentional capture modulates perceptual sensitivity. Psychonomic Bulletin \& Review. 2004; 11:551-554. [PubMed: 15376809]

Theeuwes J, Olivers CNL, Belopolsky AV. Stimulus-driven capture and contingent capture. Wiley Interdisciplinary Reviews: Cognitive Science. 2010; 1:872-881.

Treisman AM, Gelade G. A feature-integration theory of attention. Cognitive Psychology. 1980; 12:97-136. [PubMed: 7351125]

Tsotsos JK, Culhane S, Wai WK, Lai Y. Modeling visual attention via selective tuning. Artificial Intelligence. 1995; 78:507-545.

Turatto M, Galfano G. Attentional capture by color without any relevant attentional set. Perception \& Psychophysics. 2001; 63:286-297. [PubMed: 11281103]

Watson A, Pelli D. QUEST: A Bayesian adaptive psychometric method. Perception \& Psychophysics. 1983; 33:113-120. [PubMed: 6844102]

Wichmann FA, Hill NJ. The psychometric function: I. Fitting, sampling, and goodness of fit. Perception \& Psychophysics. 2001; 63:1293-1313. [PubMed: 11800458]

Wolfe, JM. Guided Searcy 4.0: Current progress with a model of visual search. In: Gray, W., editor. Integrated models of cognitive systems. New York, NY: Oxford; 2007. p. 99-119.

Yantis S, Egeth HE. On the distinction between visual salience and stimulus-driven attentional capture. Journal of Experimental Psychology: Human Perception and Performance. 1999; 25:661-676. [PubMed: 10385983]

Yeshurun Y, Carrasco M. Attention improves or impairs visual performance by enhancing spatial resolution. Nature. 1998; 396:72-75. [PubMed: 9817201] 


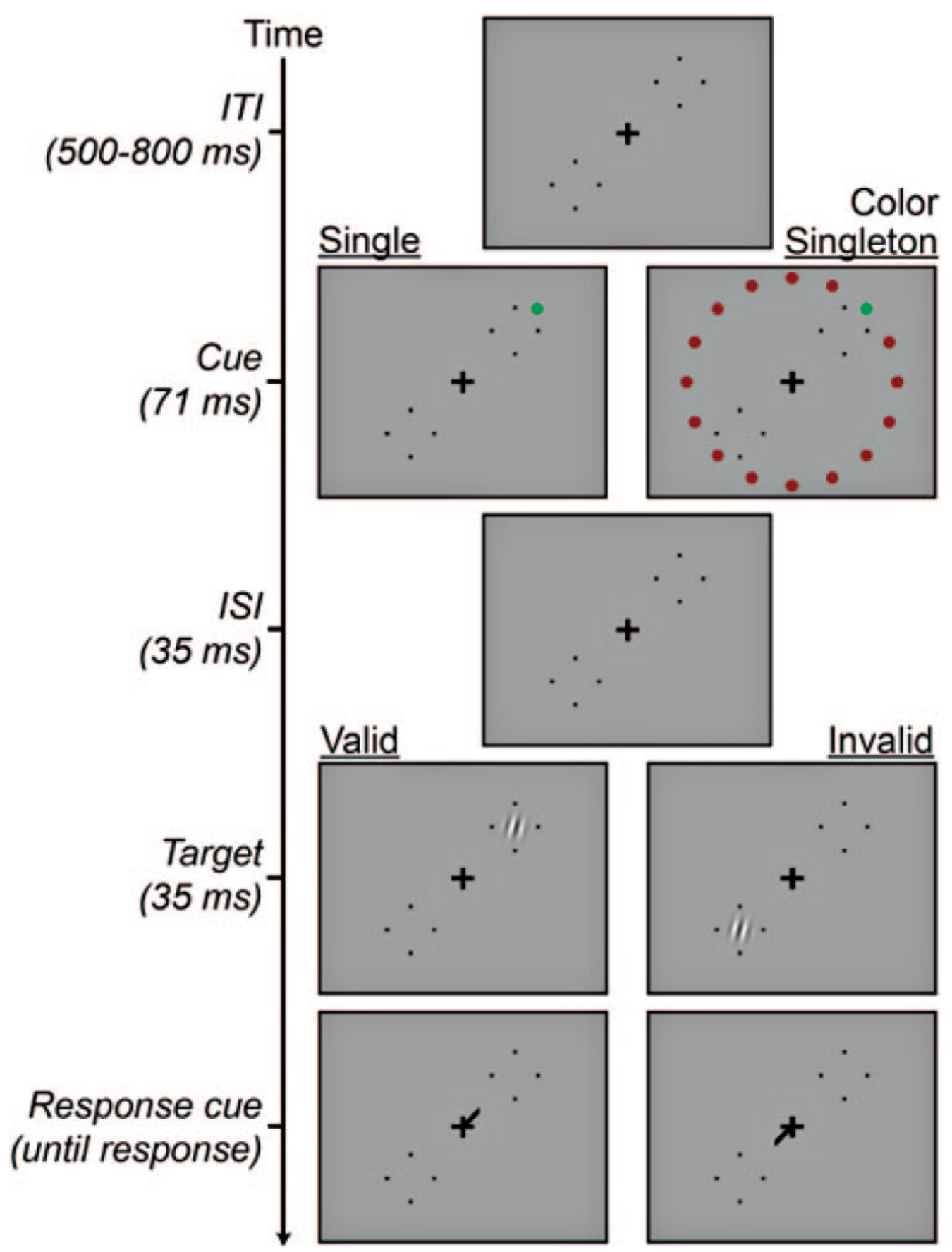

Figure 1.

Example trial sequences with green cues. 
A

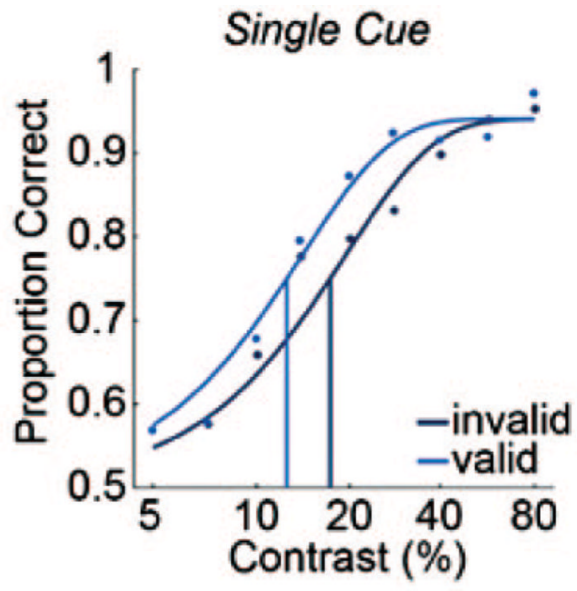

B

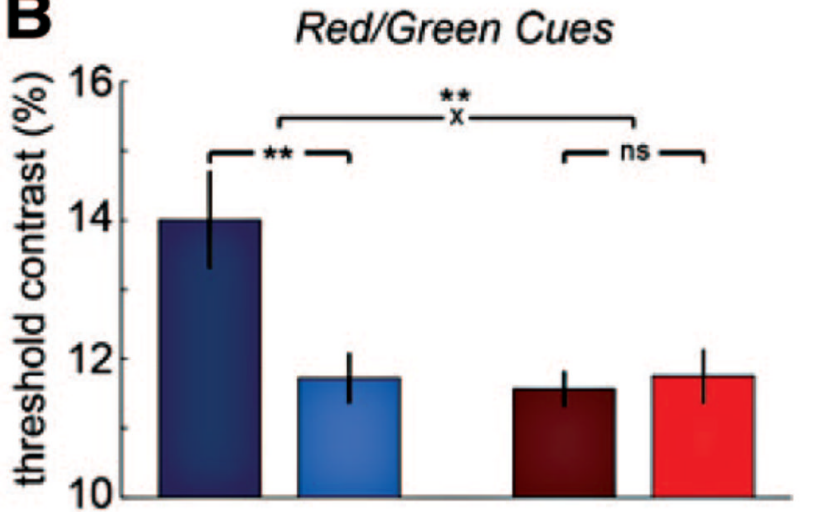

C

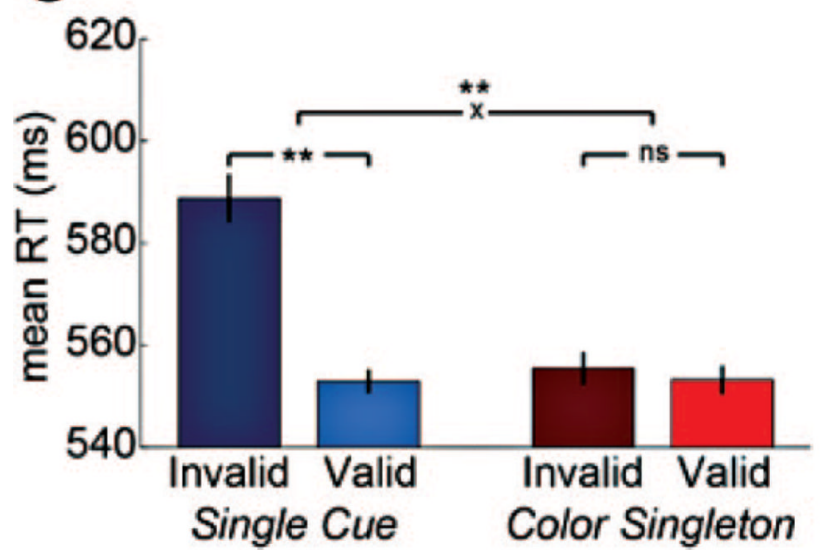

Figure 2. significant." C, RTs for Experiments 1A (left) and 1B (right).
Color Singleton

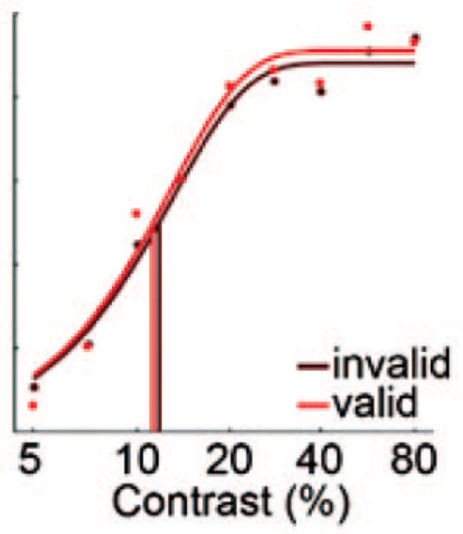

Dark/Light Cues
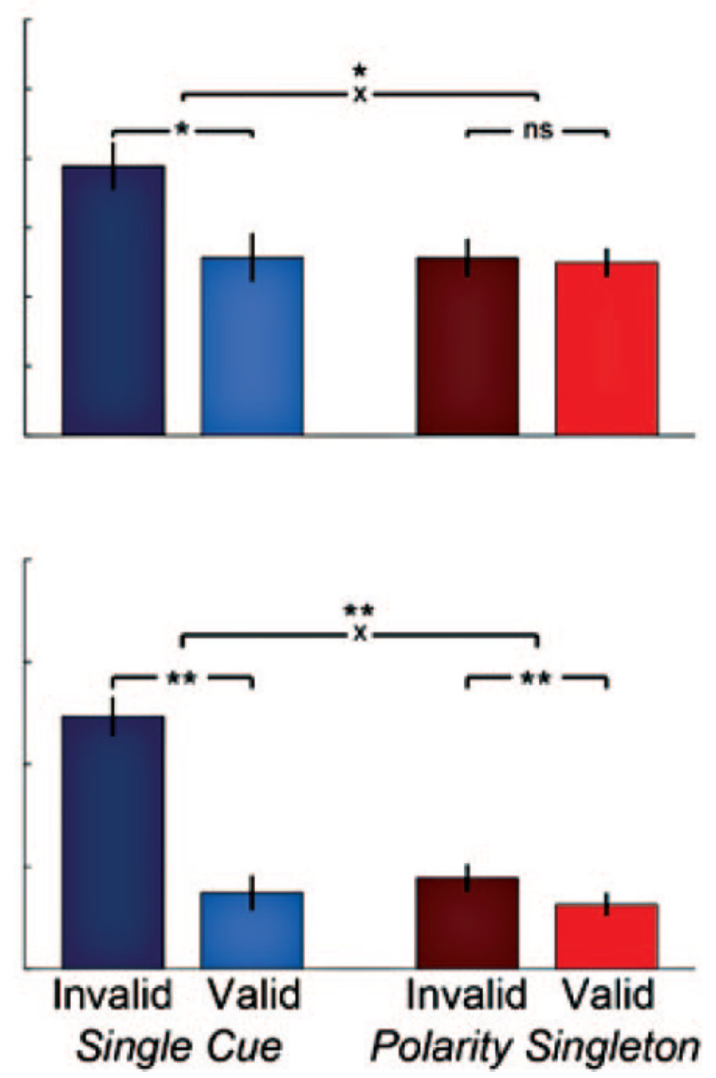

Results of Experiments 1A and 1B. A, Psychometric functions for an example observer in Experiment 1A. B, Contrast thresholds for Experiments 1A (Red/Green, left) and 1B (Dark/ Light, right). Error bars are \pm 1 SEM. Asterisks indicate $p<.05$; double asterisks indicate $p$ $<.01$. Asterisks above $x$ s are for interactions between cue validity and type. " $n s "=$ "not 
A

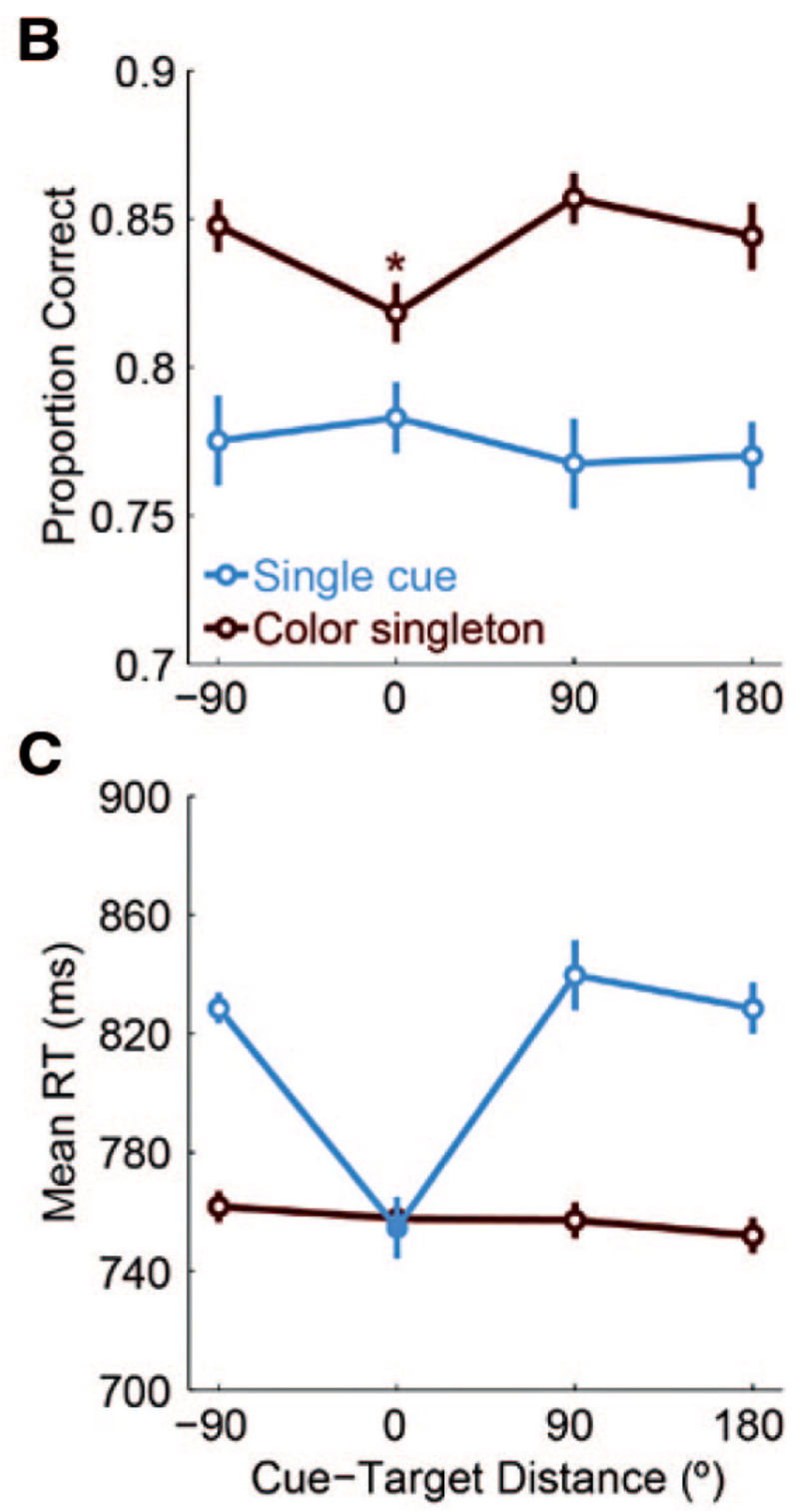

Figure 3.

Experiment 2. A, Example trial sequences with red cues and cue-target distances of $0^{\circ}$. B, Accuracy data as a function of cue-target distance for both cue types. The asterisk indicates significant difference from accuracy at $90^{\circ}$. C, RTs on correct trials. The filled symbol indicates significant difference from $180^{\circ}$ ( $p<.05$, corrected). Cue-target distance of -90 indicates that the cue and target were in the same hemifield. 
A

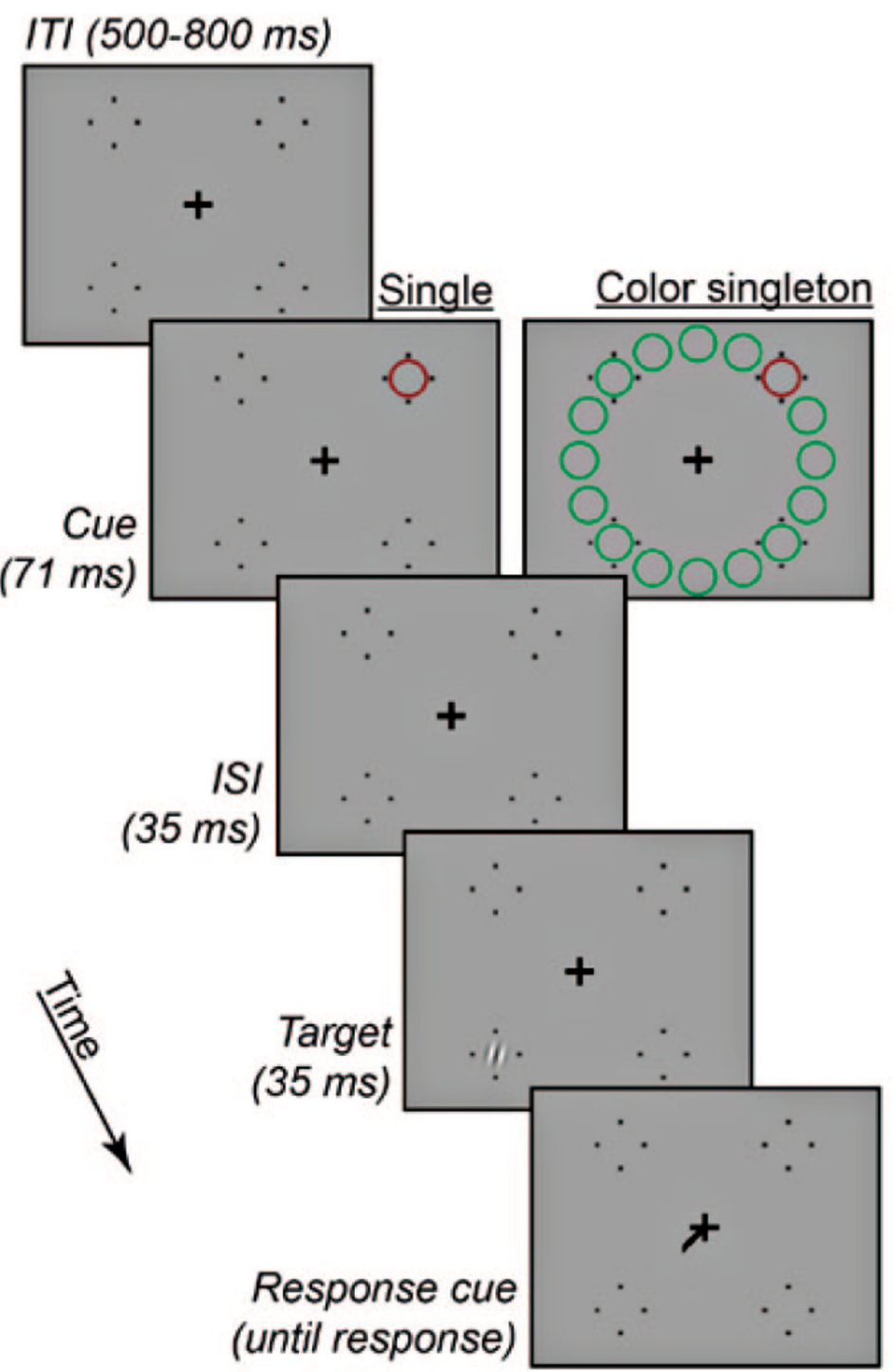

B

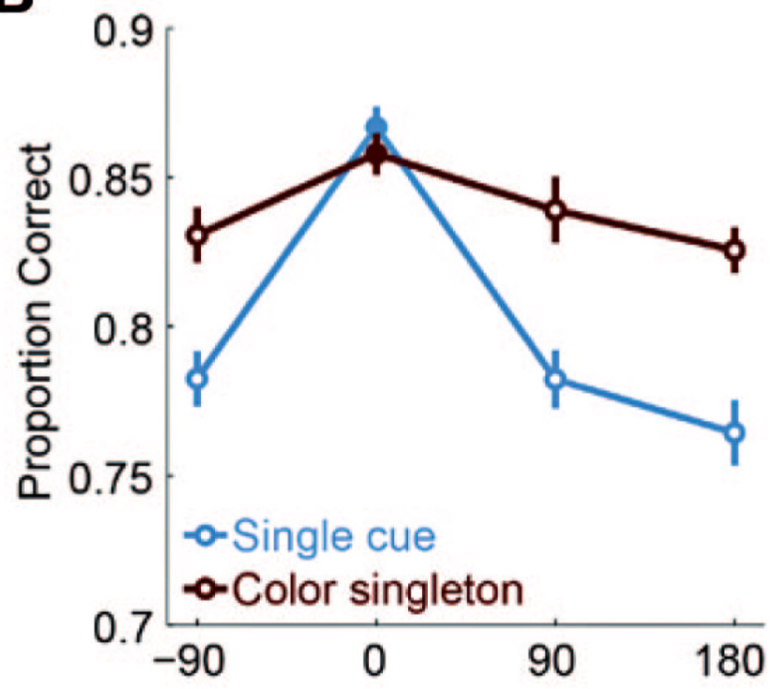

C

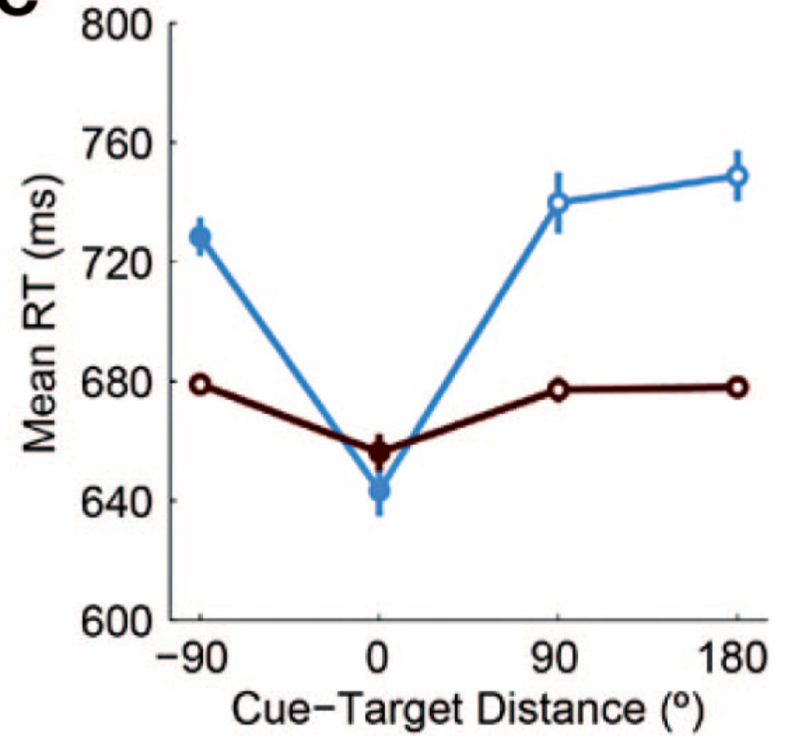

Figure 4.

Experiment 3. A, Example trial sequences with red cues and cue-target distances of $180^{\circ}$. B, Accuracy data as a function of cue-target distance for both cue types. C, RTs on correct trials. Cue-target distance of -90 indicates that the cue and target were in the same hemifield. Filled symbols indicate significant differences from $180^{\circ}(p<.05$, corrected). 
$\mathbf{A}$


$\vdots$
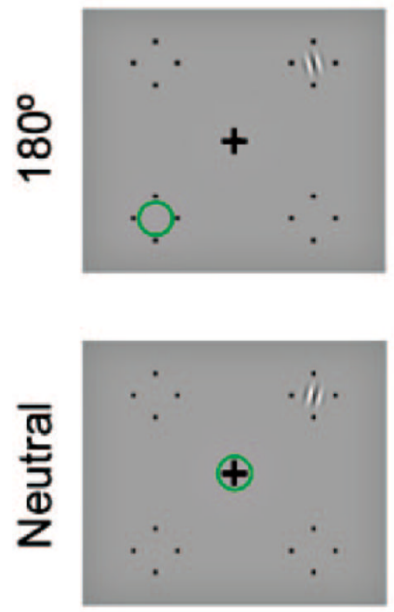
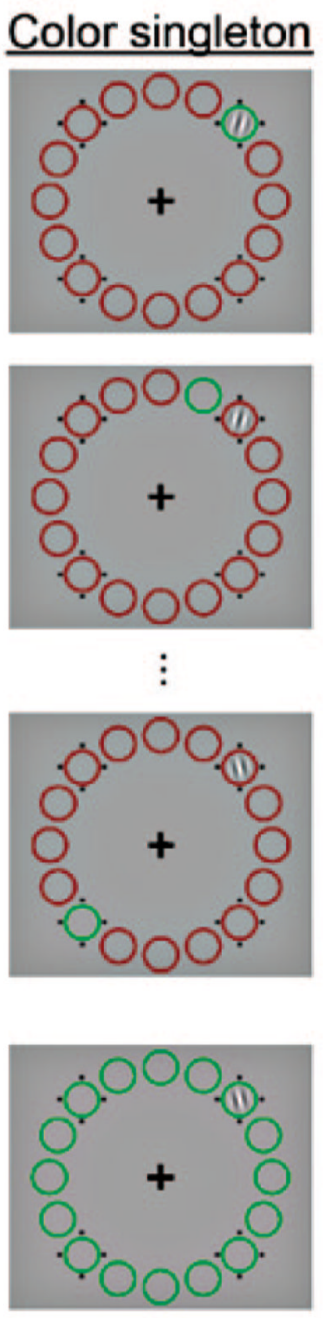

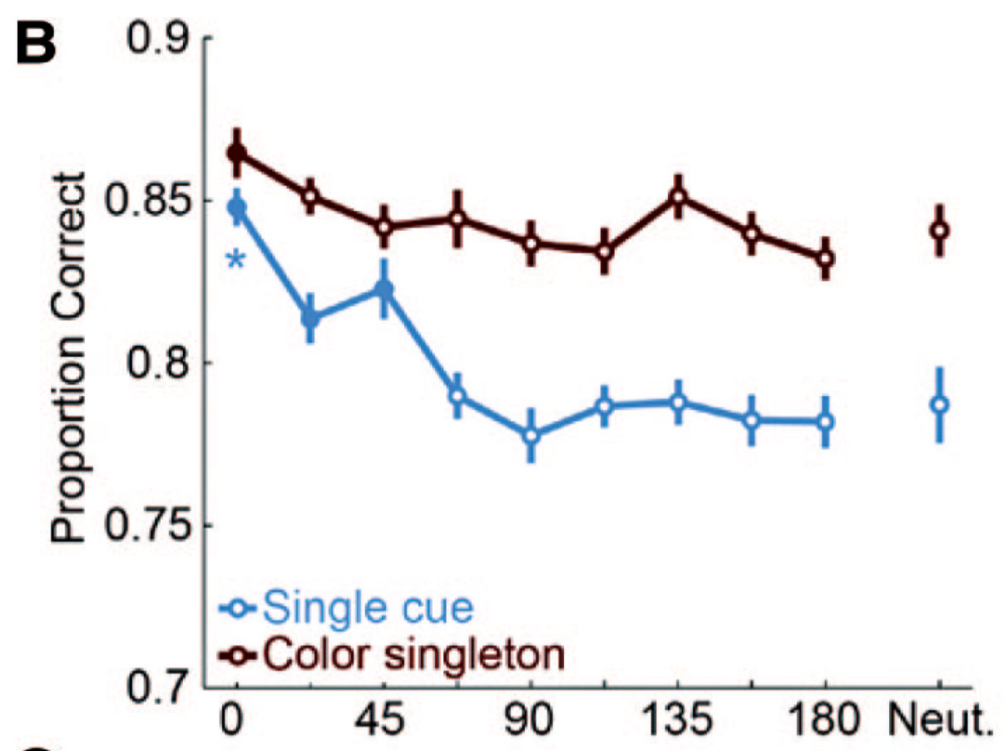

C

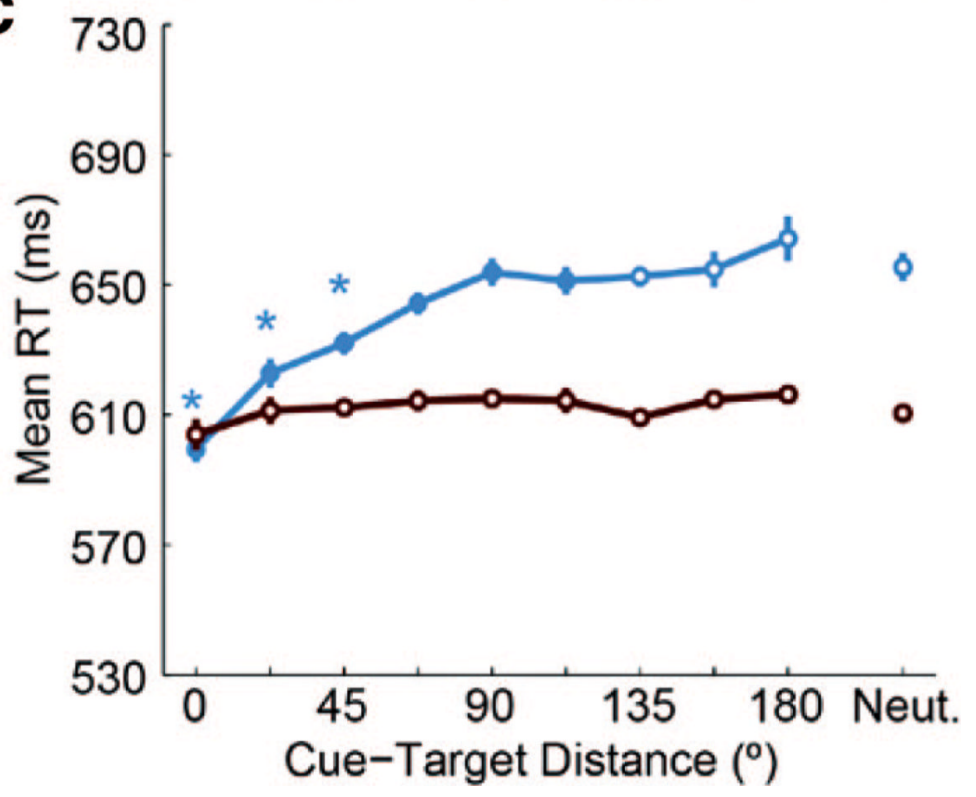

Figure 5.

Experiment 4. A, Example displays of varying cue-target distance with the cue, target, and response cue all together for the purposes of illustration (the timing was the same as in Experiment 1A). B, Accuracy data. C, RTs on correct trials. Filled symbols indicate significant differences from $180^{\circ}$; asterisks indicate significant differences from the corresponding neutral condition ( $p<.05$, corrected). 